\title{
Erratum to: New efficient method for dynamic modeling and simulation of flexible multibody systems moving in plane
}

\section{Bao Rong • Xiaoting Rui · Guoping Wang · Fufeng Yang}

Published online: 18 May 2010

(C) Springer Science+Business Media B.V. 2010

\section{Erratum to: Multibody Syst Dyn \\ DOI 10.1007/s11044-010-9196-9}

The sentence following Equation (64) is incorrectly presented. The correct presentation should be:

From (63), the transfer equation of a smooth pin hinge whose outboard body is flexible body with one inboard and one outboard points moving in plane and inboard body is a rigid body can be obtained.

Equation (66) is incorrectly presented. The correct presentation should be:

$$
\mathbf{U}_{(7+n) \times 7}=\left[\begin{array}{ccccc}
\mathbf{I}_{2} & \mathbf{O}_{2 \times 1} & \mathbf{O}_{2 \times 1} & \mathbf{O}_{2 \times 2} & \mathbf{O}_{2 \times 1} \\
\tilde{\mathbf{U}}_{21} & 0 & 0 & \tilde{\mathbf{U}}_{25} & \tilde{\mathbf{U}}_{28} \\
\mathbf{O}_{1 \times 2} & 0 & 0 & \mathbf{O}_{1 \times 2} & 0 \\
\mathbf{O}_{2 \times 2} & \mathbf{O}_{2 \times 1} & \mathbf{O}_{2 \times 1} & \mathbf{I}_{2} & \mathbf{O}_{2 \times 1} \\
\tilde{\mathbf{U}}_{51} & \mathbf{O}_{n \times 1} & \mathbf{O}_{n \times 1} & \tilde{\mathbf{U}}_{55} & \tilde{\mathbf{U}}_{58} \\
\mathbf{O}_{1 \times 2} & 0 & 0 & \mathbf{O}_{1 \times 2} & 1
\end{array}\right] .
$$

The online version of the original article can be found under doi:10.1007/s11044-010-9196-9.

B. Rong $(\bowtie) \cdot$ X.T. Rui · G.P. Wang · F.F. Yang

Institute of Launch Dynamics, Nanjing University of Science and Technology, Nanjing 210094,

P.R. China

e-mail: rongbao_nust@sina.com

X.T. Rui

e-mail: ruixt@163.net 
Equation (69) is incorrectly presented. The correct presentation should be:

$$
\mathbf{U}_{(7+n) \times 7}=\left[\begin{array}{ccccc}
\mathbf{I}_{2} & \mathbf{O}_{2 \times 1} & \mathbf{O}_{2 \times 1} & \mathbf{O}_{2 \times 2} & \mathbf{O}_{2 \times 1} \\
\mathbf{O}_{1 \times 2} & 1 & 0 & \mathbf{O}_{1 \times 2} & 0 \\
\mathbf{O}_{1 \times 2} & 0 & 1 & \mathbf{O}_{1 \times 2} & 0 \\
\mathbf{O}_{2 \times 2} & \mathbf{O}_{2 \times 1} & \mathbf{O}_{2 \times 1} & \mathbf{I}_{2} & \mathbf{O}_{2 \times 1} \\
\tilde{\mathbf{U}}_{51} & \tilde{\mathbf{U}}_{53} & \tilde{\mathbf{U}}_{54} & \tilde{\mathbf{U}}_{55} & \tilde{\mathbf{U}}_{58} \\
\mathbf{O}_{1 \times 2} & 0 & 0 & \mathbf{O}_{1 \times 2} & 1
\end{array}\right] .
$$

Equation (73) is incorrectly presented. The correct presentation should be:

$$
\mathbf{U}_{7 \times(7+n)}=\left[\begin{array}{cccc}
\mathbf{I}_{3} & \mathbf{O}_{3 \times 3} & \mathbf{U}_{13} & \mathbf{O}_{3 \times 1} \\
\mathbf{O}_{3 \times 3} & \mathbf{I}_{3} & \mathbf{O}_{3 \times n} & \mathbf{O}_{3 \times 1} \\
\mathbf{O}_{1 \times 3} & \mathbf{O}_{1 \times 3} & \mathbf{O}_{1 \times n} & 1
\end{array}\right], \quad \mathbf{U}_{13}=\left[\begin{array}{c}
\mathbf{O}_{1 \times n} \\
\mathbf{O}_{1 \times n} \\
\Phi_{O}^{\prime}
\end{array}\right] .
$$

